\title{
Magnetism in Carbon Nanoscrolls: Quasi-Half-Metals and Half-Metals in Pristine Hydrocarbons
}

\author{
Lin Lai', Jing Lu,2 $(\varangle)$, Lu Wang', Guangfu Luo', Jing Zhou', Rui Qin', Yu Chen', Hong Li', Zhengxiang Gao $(\square)$, \\ Guangping $\mathrm{Li}^{3}$, Wai Ning $\mathrm{Mei}^{2}$, Yutaka Maeda ${ }^{4,5}$, Takeshi Akasaka ${ }^{6}$, and Stefano Sanvito ${ }^{7}$ \\ ${ }^{1}$ State Key Laboratory for Mesoscopic Physics and Department of Physics, Peking University, Beijing 100871, China \\ ${ }^{2}$ Department of Physics, University of Nebraska at Omaha, Omaha, Nebraska 68182-0266, USA \\ ${ }^{3}$ SICAS Center, Lee Hall, SUNY Oneonta, Oneonta, NY 13820, USA \\ ${ }^{4}$ Department of Chemistry, Tokyo Gakugei University, Tokyo 184-8501, Japan \\ ${ }^{5}$ PRESTO, Japan Science and Technology Agency, 4-1-8 Honcho Kawagushi, Saitama, Japan \\ ${ }^{6}$ Center for Tsukuba Advanced Research Alliance, University of Tsukuba, Ibaraki 305-8577, Japan \\ ${ }^{7}$ School of Physics and CRANN, Trinity College, Dublin 2, Ireland \\ Received: 15 July 2009 / Revised: 18 August 2009 / Accepted: 19 August 2009 \\ CTsinghua University Press and Springer-Verlag 2009. This article is published with open access at Springerlink.com
}

\begin{abstract}
A magnetic ground state is revealed for the first time in zigzag-edged carbon nanoscrolls (ZCNSs) from spinunrestricted density functional theory calculations. Unlike their flat counterpart—zigzag-edged carbon nanoribbons, which are semiconductors with spin-degenerate electronic structure-ZCNSs show a variety of magnetic configurations, namely spin-selective semiconductors, metals, semimetals, quasi-half-metals, and half-metals. To the best of our knowledge, this is the first discovery of quasi-half-metals and half-metals in a pure hydrocarbon without resort to an external electric field. In addition, we calculated the spin-dependent transportation of the semiconducting ZCNSs with 12 and 20 zigzag chains, and found that they are $13 \%$ and $17 \%$ at the Fermi level, respectively, suggesting that ZCNS can be an effective spin filter.
\end{abstract}

\section{KEYWORDS}

Carbon nanoscrolls, quasi-half-metals, half-metals, graphene, spin-selective, spin filter

Graphene has attracted substantial interest because of its potential applications in nanoelectronics [111]. Graphene can be fabricated into a new kind of quasi-one-dimensional material known as graphene nanoribbons (GNRs). Spin-unrestricted density functional theory (DFT) calculations predicted that H-terminated GNRs with zigzag edges (ZGNRs) have a magnetic ground state with ferromagnetic (FM) ordering at each zigzag edge and antiparallel spin orientation between the two edges [4, 11]. Because the two spins occupy two staggered sublattices and are mirror symmetrical, narrow ZGNRs $(<100 \AA)$ are always semiconductors, with completely spindegenerate electronic structures $[4,6,10]$. Such spin degeneracy in the electronic structure can be lifted by applying a transverse in-plane external electric field $[4,11]$ or by employing $\mathrm{NO}_{2}$ groups on one edge and $\mathrm{CH}_{3}$ groups on the other edge, since both of these

Address correspondence to Jing Lu, jinglu@pku.edu.cn; Zhengxiang Gao, zxgao@pku.edu.cn 
two approaches break the spin mirror symmetry of ZGNRs [9]. Correspondingly, ZGNRs are transformed into a spin-selective semiconductor or even a halfmetal and have potential applications in spintronics. So far, it is unclear whether a half-metal is available in a pure hydrocarbon.

Carbon nanoscrolls (CNSs) can be regarded as an open scrolled product of GNRs, and are traditionally synthesized by ultrasonication of graphene [1214]. Very recently, a simple and effective method has been developed to fabricate high-quality CNSs by using isopropyl alcohol solution to roll up monolayer graphene predefined on $\mathrm{SiO}_{2} / \mathrm{Si}$ substrates [15]. From spin-restricted DFT calculations within the local density approximation (LDA), CNSs are predicted to be even lower in total energy than their respective GNRs if the inner radius of the CNS is greater than $11.5 \AA$, due to the large flexibility of carbon nanoribbons [16]. They have potential applications in hydrogen storage and energy storage in supercapacitors and batteries [12, 17-20]. Spin-restricted DFT calculations predicted that $\mathrm{H}$-terminated zigzag-edged carbon nanoscrolls (ZCNSs) are metals or semimetals with two flat bands at the Fermi level $\left(E_{f}\right)[16,21]$, which results in a very large density of states at $E_{\mathrm{f}}$. In principle, such an infinitesimally small on-site Coulomb repulsion could make H-terminated ZCNSs magnetic according to Stoner theory.

From spin-restricted DFT calculations, the electronic structure of ZGNRs is also characterized by a set of doubly degenerate flat bands at $E_{\mathrm{f}}[4,11]$. Inspired by the theoretical discovery of the magnetic ground state in ZGNRs from spin-unrestricted DFT calculations, here we reexamine the electronic structure of H-passivated ZCNSs within the local spin density approximation (LSDA). We find that ZCNSs do show a magnetic ground state with an FM ordering at each zigzag edge. The two edges are usually antiferromagnetically (AFM) coupled as in the ZGNR cases. Remarkably, spin degeneracy in the electronic structure has been lifted in ZCNSs, and ZCNSs become spin-selective semiconductors, metals or even quasi-half-metals and half-metals. Spinselective conductance is found when the quantum transport properties of ZCNSs are studied by combining LSDA and the non-equilibrium Green's function (NEGF) method.

Following the previous work (Fig. 1) [16], we generate the initial geometric structure of a CNS by rolling up a graphene nanoribbon (GNR) into a truncated Archimedean-type spiral, which is defined by the equation $r=d \theta / 2 \pi+c$, where $r$ and $\theta$ are the usual cylindrical coordinates. The nonzero constant $c$ is the initial inner radius of the CNS, and the interlayer spacing $d$ is set to $3.4 \AA$. We use $(n, m)$ ZCNS to denote a ZCNS, where $n$ is the total number of zigzag chains in the ZCNS and $m$ is the roughly estimated number of zigzag chains in the overlap region.

Geometry optimization and electronic band calculations were performed with the first-principles package, SIESTA $[22,23]$. We adopt the double- $\zeta$ plus polarization basis set (DZP) and norm-conserving pseudopotentials of the Troullier-Martins type [24]. A mesh cutoff of 120 and 200 Ry is employed for the integration of the real space grid in the geometry optimization and static total energy calculation, respectively. A $1 \times 1 \times 12 k$-point grid of MonkhorstPack type is employed to sample the Brillouin zone [25]. The nearest inter-atomic distance between the H-terminated CNS and its images is kept larger than $8 \AA$ in our supercell model. We optimize both the atomic positions and lattice parameter of the CNSs.

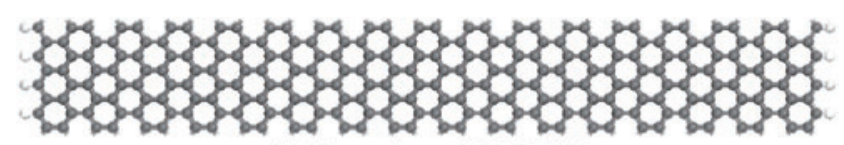

(a) H-terminated 32-ZGNR

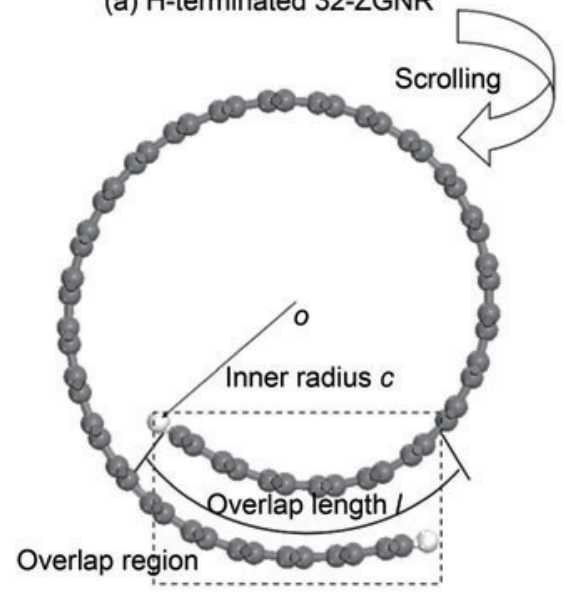

(b) $(32,5)$ ZCNS

Figure 1 Illustration of the definition of the parameters of a CNS. Gray and white balls denote $\mathrm{C}$ and $\mathrm{H}$ atoms, respectively 
The convergence criterion for the total force on each atom is $0.05 \mathrm{eV} / \AA$. A periodic potential of sawtooth type perpendicular to the axis direction of the ZCNS is used to simulate the external electric field in a supercell. The quantum transport properties are calculated with the ab initio Smeagol code [26, 27], which is based on an NEGF + LSDA approach to simulate open systems. The single- $\zeta$ plus polarization basis set (SZP), norm-conserving pseudopotentials of the Troullier-Martins type, and a mesh cutoff of 200 Ry are used [24].

We take fourteen ZCNSs into account and summarize the key results in Table 1. Optimized structures of six of the ZCNSs are shown in Fig. 2 and those of the remaining species are shown in Fig. S-1 in the electronic supplementary material (ESM). Spin-polarized calculations lead to negligible changes in atomic structure compared with those obtained in the spin-unpolarized calculations [16]. In general, the smaller is the size, the larger is the deformation extent from the initial structure. The optimized structures of the $(48,7),(56,3),(56,13)$, and $(60,20)$ ZCNSs slightly or moderately deviate from their initial ones, while the optimized structures of the $(12,2)$ and $(20,2)$ ZCNSs are deformed to a large extent. Nine of the fourteen ZCNSs are expected to be more stable than

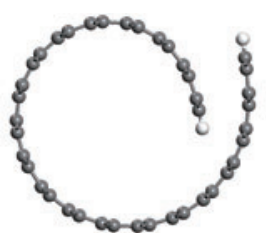

(a) $(12,2)-Z C N S$

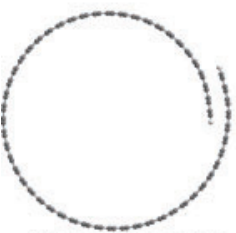

(d) $(56,3)-\mathrm{ZCNS}$

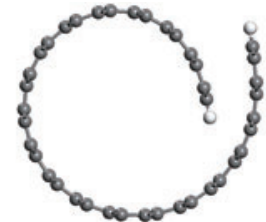

(b) $(20,2)-Z C N S$

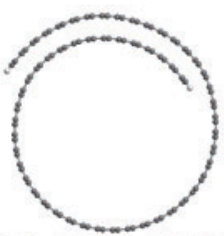

(e) $(56,13)-Z C N S$

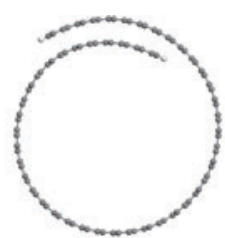

(c) $(48,7)-\mathrm{ZCNS}$

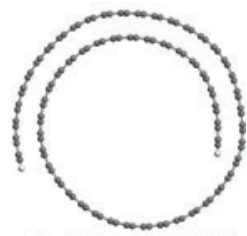

(f) $(60,20)-\mathrm{ZCNS}$
Figure 2 Optimized structures of the (a) $(12,2)$, (b) $(20,2)$, (c) $(48,7)$, (d) $(56,3)$, (e) $(56,13)$, and (f) $(60,20)$ ZCNSs. Gray and white balls represent $\mathrm{C}$ and $\mathrm{H}$ atoms, respectively

their flat counterparts since their inner diameters are greater than $11.5 \AA$ [16]. The energy gain due to spin polarization ranges from 2.4 to $185.6 \mathrm{meV}$ per edge atom. Similar to ZGNRs, the spin on each edge of ZCNSs favors an FM ordering [4]. The spins at the two edges usually have antiparallel orientation, and the FM-AFM energy differences range from 0.2 to $9.2 \mathrm{meV}$ per edge atom. The two exceptions are the $(56,13)$ - and $(60,2)$-ZCNSs, where the FM state is more stable than the corresponding AFM state by 0.5

Table 1 Inner radius $(r)$, overlap length (I), relative total energy per edge atom between the ferromagnetic $\left(E_{\mathrm{FM}}\right)$, antiferromagnetic $\left(E_{\mathrm{AFM}}\right)$, nonmagnetic states $\left(E_{\mathrm{NM}}\right)$, and spin-selective band gap in the ground state of the ZCNSS

\begin{tabular}{|c|c|c|c|c|c|c|c|}
\hline ZCNS & $r(\AA ̊)$ & I (Å) & $\begin{array}{c}E_{\mathrm{AFM}}-E_{\mathrm{NM}} \\
(\mathrm{meV})\end{array}$ & $\begin{array}{c}E_{\mathrm{FM}}-E_{\mathrm{NM}} \\
(\mathrm{meV})\end{array}$ & $\begin{array}{c}E_{\mathrm{AFM}}-E_{\mathrm{FM}} \\
(\mathrm{meV})\end{array}$ & $E_{\mathrm{g}}^{\text {smaller }}(\mathrm{eV})$ & $E_{\mathrm{g}}^{\text {larger }}(\mathrm{eV})$ \\
\hline$(12,2)$ & 2.6 & 4.3 & -10.1 & -3.9 & -6.2 & 0.19 & 0.46 \\
\hline$(20,2)$ & 5.5 & 4.3 & -185.6 & -182.4 & -3.2 & 0.05 & 0.25 \\
\hline$(32,5)$ & 8.0 & 9.3 & -42.8 & -40.5 & -2.3 & 0.11 & 0.28 \\
\hline$(40,5)$ & 11.0 & 9.3 & -13.4 & -7.4 & -6.0 & 0.30 & 0.31 \\
\hline$(48,7)^{a}$ & 12.8 & 15.7 & -90.6 & -88.6 & -2.0 & 0.01 & 0.19 \\
\hline$(56,13)$ & 13.1 & 24.9 & -20.6 & -21.1 & 0.5 & Metallic & Metallic \\
\hline$(56,8)$ & 15.0 & 17.1 & -19.2 & -16.3 & -2.9 & 0.04 & 0.10 \\
\hline$(56,3)^{b}$ & 17.0 & 7.1 & -13.1 & -3.9 & -9.2 & Semimetallic & 0.05 \\
\hline$(56,2)$ & 17.5 & 4.3 & -2.4 & 0.7 & -3.1 & Semimetallic & Semimetallic \\
\hline$(60,20)$ & 11.2 & 42.7 & -18.9 & -17.8 & -1.1 & Semimetallic & Semimetallic \\
\hline$(60,16)$ & 13.1 & 34.2 & -19.4 & -19.2 & -0.2 & 0.01 & 0.02 \\
\hline$(60,12)$ & 15.0 & 25.6 & -18.5 & -18.2 & -0.3 & 0.08 & 0.10 \\
\hline$(60,7)$ & 17.1 & 14.9 & -17.1 & -15.3 & -1.8 & 0.10 & 0.15 \\
\hline$(60,2)$ & 19.0 & 4.3 & -15.1 & -15.2 & 0.1 & Metallic & Metallic \\
\hline
\end{tabular}

${ }^{a}$ Quasi-half-metal.

${ }^{b}$ Half-metal. 
and $0.1 \mathrm{meV}$ per edge atom, respectively.

The spin degeneracies in electronic structure near the Fermi level $\left(E_{\mathrm{f}}\right)$ of the AFM state are lifted in all the fourteen ZCNSs, while those far away from $E_{\mathrm{f}}$ remain intact. We present the ground-state electronic structures of the (12,2)-, (20,2)-, (48,7)-, $(56,3)-,(56,13)-$, and $(60,20)-Z C N S s$ in Fig. 3 and those of the remaining species are shown in Fig. S-2 in the ESM. The electronic properties of the ZCNSs are summarized in Table 1 . Nine ZCNSs are spinselective semiconductors, and the difference in the band gap for different spin channels is apparent, except for the $(40,5)$ - and $(60,16)$-ZCNSs. In particular, the smaller band gap is merely $0.01 \mathrm{eV}$, while the larger one is $0.19 \mathrm{eV}$ in the $(48,7)-\mathrm{ZCNS}$, making this material a quasi-half-metal. The $(56,2)-$, $(60,20)-$, and $(60,16)-Z C N S s$ are semi-metals due to an overlap between their conduction and valence bands. Remarkably, the $(56,3)$-ZCNS is a half-metal with one conductive spin channel and a band gap of $0.05 \mathrm{eV}$ in another spin channel. To the best of our knowledge, the $(48,7)$-ZCNS and $(56,3)$-ZCNS are the first quasihalf-metal and half-metal, respectively, existing in a hydrocarbon without resort to an external electric field. The $(56,13)$ - and $(60,2)$-ZCNSs are metals because they have FM ground states and the FM states of ZCNSs are always metallic, as the case of ZGNRs [28].

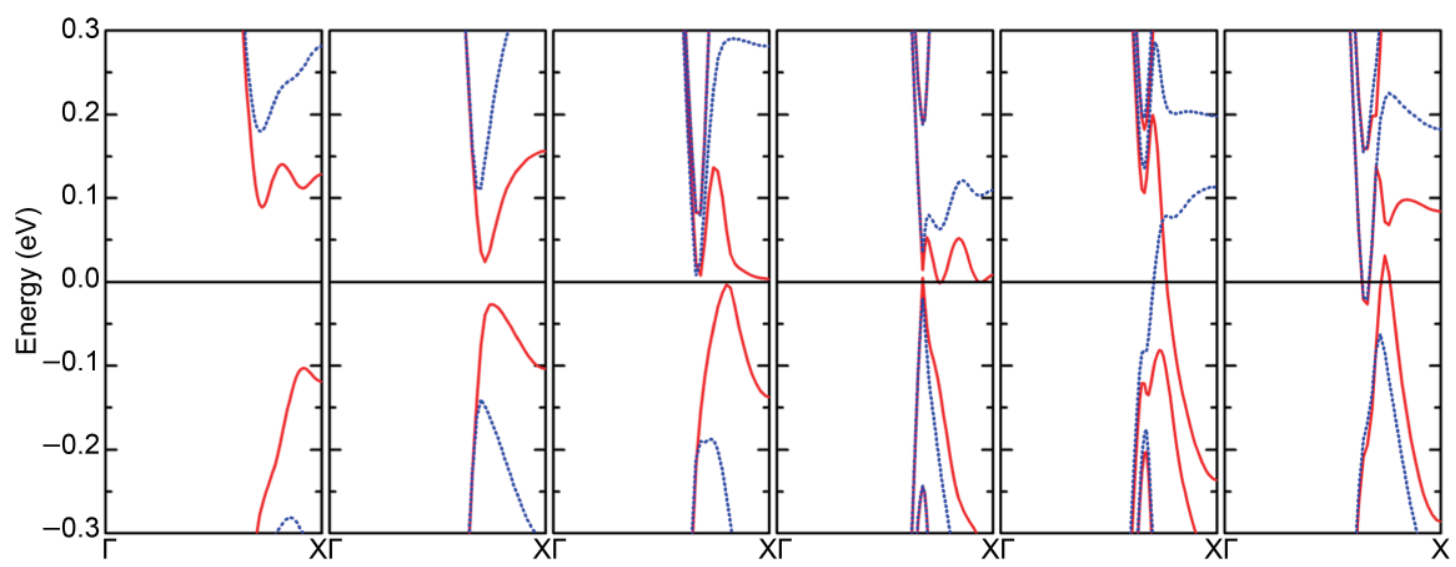

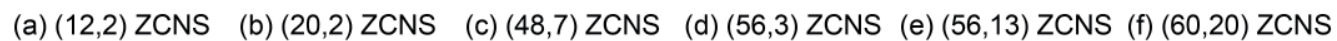

Figure 3 Spin-resolved electronic structures of the (a) (12,2)-, (b) $(20,2)-$, (c) $(48,7)-$, (d) (56,3)-, and (e) $(60,20)-Z C N S$. The solid red lines and dotted blue lines represent the bands of the spin-up and spin-down states, respectively. The Fermi level is set to zero

By analyzing the data in Table 1, we find the following rules that apply to the electronic structures of the large $(56, m)$ and $(60, m)$ ZCNSs: the band gap is opened for moderate $m$ values, and it is closed for larger and smaller $m$ valves. The band gaps of both spins of the $(60, m)$ ZCNS decrease with increasing $m$ for $m=7-20$. In a flat ZGNR, the two spins of the AFM state of a ZGNR occupy two staggered sublattices and are chiefly mirror-symmetrically distributed on the two edges. This is responsible for the opening of the band gap and the spin degeneracy of the band structure of a ZGNR. Similar to a ZGNR [4], the two spins of the AFM state of a ZCNS also occupy two staggered sublattices and are chiefly distributed on the two edges (Fig. 4). However, the two spins have no mirror symmetry, and this could

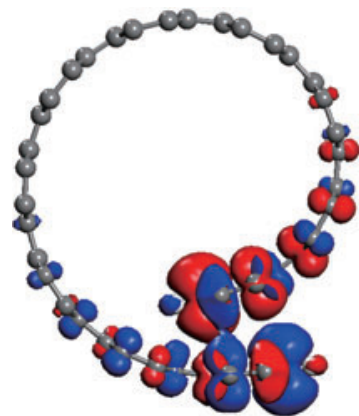

(a)

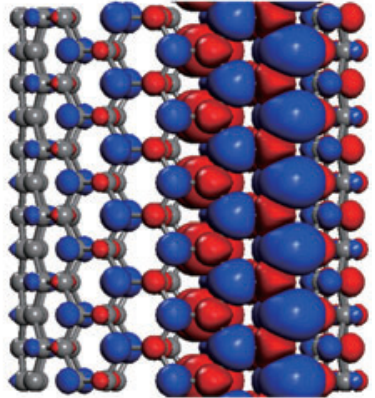

(b)
Figure 4 Spin density in the ground state of the (20,2)-ZCNS with an isovalue of $0.003 \mathrm{e} / \AA^{3}$ : (a) top view and (b) side view. The red and blue represent the up and down spins, respectively

reduce the band gap and lift the spin degeneracy in the electronic structure of a ZCNS. The larger is the deviation of the geometry of a ZCNS from its flat counterpart, the smaller is the band gap of a 
ZCNS. Xie et al. measured the electrical transport of a well-stacked CNS with an outer diameter of $5 \AA$ [15]. Such a CNS has a larger ratio of $m$ to $n$. If it has a zigzag edge, it should be metallic. If it has an armchair edge, its band gap is also very small and approaches zero, based on our previous DFT calculations [16]. In both cases, this CNS was predicted to have a good conductivity and weak gate dependence. Experimentally, Xie et al. [15] found that it does indeed have low resistance and is weakly gate-dependent. This supports our other electronic structure predictions for CNSs. As for the closure of the band gap at smaller $m$, we attribute this to the stronger interaction between the two magnetic edges because the distance between the two edges is apparently smaller in the case with less overlap. Because the two spins are asymmetrical, the band gaps are not closed simultaneously. Half-metallicity appears as a result of different closing speeds of the band gaps for the two spins. In principle, halfmetallicity of ZCNS should occur before the band gaps for the two spins are closed. Namely, this state should lie between semiconducting and metallic ZCNSs. The $(56,3)$ ZCNS is such an example. Its larger- $m$ neighbor $(56,8)$ ZCNS is a semiconductor, while the smaller- $m$ neighbor $(56,2)$ ZCNS is a metal.

Spontaneous spin polarization in a onedimensional infinite system at finite temperature is forbidden. However, it can occur in a nanoscale material assisted by the enhanced anisotropy on substrates [4]. Moreover, as pointed out by a tightbinding model calculation [29], a perturbation (e.g., by bias or temperature) on the order of the band gap can lead to an instability of the spin-polarized state of ZGNRs and make the system become unpolarized. Therefore, half-metallicity and quasi-half-metallicity of ZCNSs should exist at a low bias and low temperature. From the small band gap of $0.05 \mathrm{~V}$ in the half-metallic $(56,3)$ ZGNR, we can estimate the threshold bias for loss of half-metallicity to be as small as about $0.05 \mathrm{~V}$ and the threshold temperature to be about $573 \mathrm{~K}$.

A spin-selective band structure is expected to cause a spin-dependent transport. We use a two-probe system (shown in Fig. 5(a)) to calculate the quantum transport property of two small-sized ZCNSs. The left and right electrodes are semi-infinite ZCNSs identical to that in the scattering region. Since unpolarized ZCNSs are always metallic, we can force the spin state of the ZCNS electrode to be unpolarized in order to create metallic electrodes. The resulting spin-polarized conductances of the AFM (12,2)- and (20,2)-ZCNSs are shown in Figs. 5(b) and 5(c), respectively. The conductance at $E_{\mathrm{f}}$ of the AFM $(12,2)$-ZCNS is $1.65 G_{0}$ and $1.27 G_{0}$ for the spin-up and spin-down channels, respectively, and that of the AFM $(20,2)-Z C N S$ is $1.43 G_{0}$ and $1.01 G_{0}$ for the spin-up and spin-down channels, respectively. The spin transport polarization $(\xi)$ can be measured through the expression,

$$
\xi=\left|\frac{G^{\text {up }}-G^{\text {down }}}{G^{\text {up }}+G^{\text {down }}}\right|
$$

where $G^{\text {down }}$ and $G^{\text {up }}$ represent the conductance of the spin-down and spin-up channels, respectively. The $\operatorname{AFM}(12,2)$ - and $(20,2)-Z C N S s$ have $\xi$ values of $13 \%$ and $17 \%$ at $E_{\mathrm{f}}$, respectively, which are comparable to

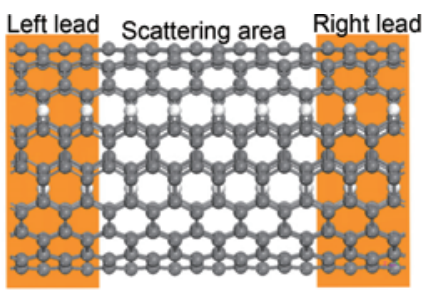

(a)

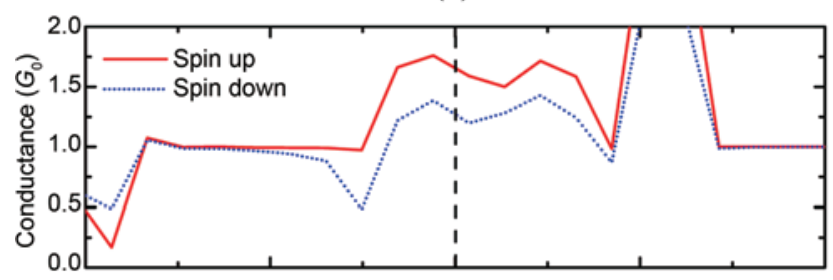

(b)

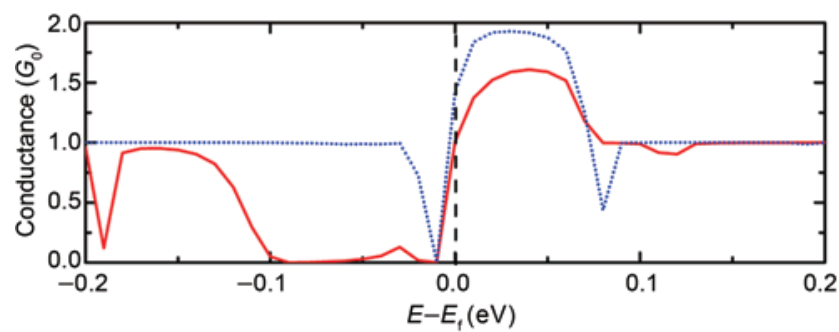

(c)

Figure 5 (a) Two-probe model of the (12,2)-ZCNS. The scattering region contains five unit cells of the ZCNS. Zero-bias conductances of the antiferromagnetic (b) $(12,2)-Z C N S$ and (c) $(20,2)$ ZCNS coupled to the homogeneous nonmagnetic ZCNS electrode. The solid red line and dotted blue line represent the conductance of the spin-up and spin-down states, respectively 
that of the boron-doped FM ZGNR with three zigzag carbon chains obtained in a previous DFT calculation [28]. We expect that ZCNSs can serve as an effective spin filter.

Finally, we examine the effects of an electric field on the band structures of the ZCNSs. In contrast to ZGNRs [4, 8], in general the application of an electric field cannot close the band gaps of the semiconducting ZCNSs. One exception is the $(40,5)-Z C N S$, where the spin degeneracy is merely slightly lifted. The effects of the electric field depend on its strength and direction. When an electric field is applied along the $x$-direction (shown in Fig. 6), the band gap decreases faster in the spin-up orientation than in the spin-down orientation. When the strength of the electric field is in the range $0.25-0.30 \mathrm{eV} / \AA$, the band gap in the spin-up direction is completely closed, whereas that in the spin-down direction remains unclosed, as shown in Fig. 6. Hence, the semiconducting $(40,5)$-ZCNS is driven to be a halfmetal.

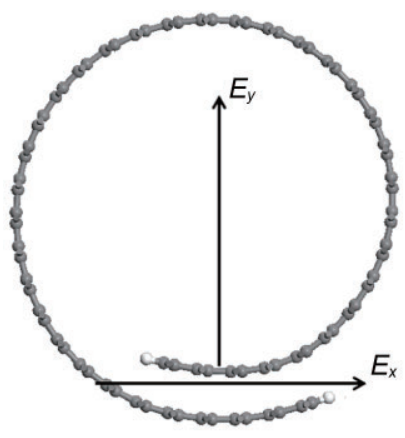

(a)

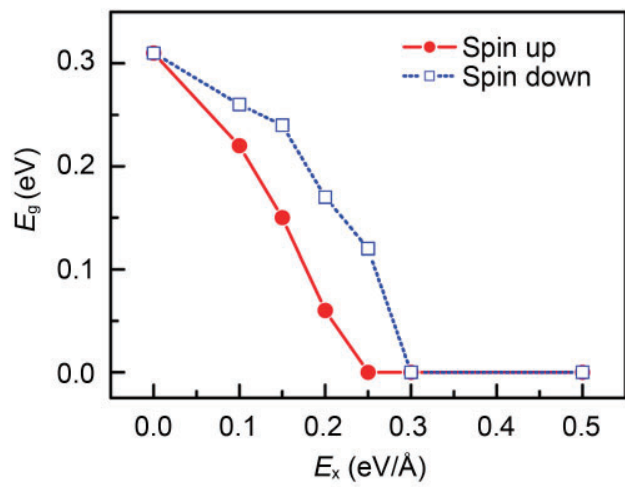

(b)

Figure 6 Band gaps of the $(40,5)$-ZCNS as a function of the external electric field (along the $x$-direction) in the spin-up and spin-down directions

In conclusion, from spin-polarized density functional theory calculations magnetism is found for the first time to be present in zigzag-edged carbon nanoscrolls. The electronic properties of zigzag carbon nanoscrolls range from spin-selective semiconductors, metals to quasi-half-metals and halfmetals, quite different from the flat zigzag-edged carbon nanoribbons. To the best of our knowledge, this is the first discovery of quasi-half-metallicity and half-metallicity in a pure hydrocarbon without resort to an external electric field. Further work is underway to reveal the connection between geometric structure and these novel magnetic configurations. Furthermore, spin-selective transport is found in semiconducting zigzag carbon nanoscrolls. These findings suggest potential applications of ZCNSs as spin filters and other nanoscale devices in spintronics. In view of the fact that magnetism in nanoscale graphene has been experimentally verified $[30,31]$, we hope our theoretical calculations will stimulate experimental work to confirm the existence of magnetism in carbon nanoscrolls.

\section{Acknowledgements}

This work was supported by the National Natural Science Foundation of China (NSFC) (Grant Nos. 10774003, 10474123, 10434010, 90606023, and 20731160012), National 973 Projects (No. 2007CB936200, Ministry of Science and Technology (MOST) of China), Program for New Century Excellent Talents in University of Ministry of Education (MOE) of China, the Grant-in-Aid for National Research Grid Initiative (NAREGI) Nanoscience Project from the Ministry of Education, Sports, Culture, Science and Technology (MEXT) of Japan, the Kurata Memorial Hitachi Science and Technology Foundation, and Nebraska Research Initiative (No. 4132050400) of USA.

Electronic Supplementary Material: Optimized geometries and electronic structures of the other eight zigzag-edged carbon nanoscrolls are available in the online version of this article at http://dx.doi.org/10.1007/s12274-009-9081-0 and are accessible free of charge.

\section{References}

[1] Geim, A. K.; Novoselov, K. S. The rise of graphene. Nat. Mater. 2007, 6, 183-191.

[2] Novoselov, K. S.; Geim, A. K.; Morozov, S. V.; Jiang, D.; Katsnelson, M. I.; Grigorieva, I. V.; Dubonos, S. V.; Firsov, A. A. Two-dimensional gas of massless Dirac fermions in 
graphene. Nature 2005, 438, 197-200.

[3] Avouris, P.; Chen, Z. H.; Perebeinos, V. Carbon-based electronics. Nat. Nanotechnol. 2007, 2, 605-615.

[4] Son, Y. W.; Cohen, M. L.; Louie, S. G. Energy gaps in graphene nanoribbons. Phys. Rev. Lett. 2006, 97, 216803.

[5] Li, X. L.; Wang, X. R.; Zhang, L.; Lee, S. W.; Dai, H. J. Chemically derived, ultrasmooth graphene nanoribbon semiconductors. Science 2008, 319, 1229-1232.

[6] Barone, V.; Hod, O.; Scuseria, G. E. Electronic structure and stability of semiconducting graphene nanoribbons. Nano Lett. 2006, 6, 2748-2754.

[7] Han, M. Y.; Ozyilmaz, B.; Zhang, Y. B.; Kim, P. Energy band-gap engineering of graphene nanoribbons. Phys. Rev. Lett. 2007, 98, 206805.

[8] Hod, O.; Barone, V.; Peralta, J. E.; Scuseria, G. E. Enhanced half-metallicity in edge-oxidized zigzag graphene nanoribbons. Nano Lett. 2007, 7, 2295-2299.

[9] Kan, E. J.; Li, Z. Y.; Yang, J. L.; Hou, J. G. Half-metallicity in edge-modified zigzag graphene nanoribbons. J. Am. Chem. Soc. 2008, 130, 4224-4225.

[10] Yang, L.; Park, C. H.; Son, Y. W.; Cohen, M. L.; Louie, S. G. Quasiparticle energies and band gaps in graphene nanoribbons. Phys. Rev. Lett. 2007, 99, 186801.

[11] Son, Y. W.; Cohen, M. L.; Louie, S. G. Half-metallic graphene nanoribbons. Nature 2006, 444, 347-349.

[12] Viculis, L. M.; Mack, J. J.; Kaner, R. B. A chemical route to carbon nanoscrolls. Science 2003, 299, 1361.

[13] Roy, D.; Angeles-Tactay, E.; Brown, R. J. C.; Spencer, S. J.; Fry, T.; Dunton, T. A.; Young, T.; Milton, M. J. T, Synthesis and raman spectroscopic characterisation of carbon nanoscrolls. Chem. Phys. Lett. 2008, 465, 254-257.

[14] Savoskin, M. V.; Mochalin, V. N.; Yaroshenko, A. P.; Lazareva, N. I.; Konstantinova, T. E.; Barsukov, I. V.; Prokofiev, I. G. Carbon nanoscrolls produced from acceptor-type graphite intercalation compounds. Carbon 2007, 45, 2797-2800.

[15] Xie, X.; Ju, L.; Feng, X. F.; Sun, Y. H.; Zhou, R. F.; Liu, K.; Fan, S. S.; Li, Q. Q.; Jiang, K. L. Controlled fabrication of high-quality carbon nanoscrolls from monolayer graphene. Nano Lett. 2009, 9, 2565-2570.

[16] Chen, Y.; Lu, J.; Gao, Z. X. Structural and electronic study of nanoscrolls rolled up by a single graphene sheet. J. Phys. Chem. C 2007, 111, 1625-1630.

[17] Braga, S. F.; Coluci, V. R.; Baughman, R. H.; Galvao, D. $\mathrm{S}$. Hydrogen storage in carbon nanoscrolls: An atomistic molecular dynamics study. Chem. Phys. Lett. 2007, 441, 78-82.

[18] Braga, S. F.; Coluci, V. R.; Legoas, S. B.; Giro, R.; Galva, D. S.; Baughman, R. H. Structure and dynamics of carbon nanoscrolls. Nano Lett. 2004, 4, 881-884.

[19] Coluci, V. R.; Braga, S. F.; Baughman, R. H.; Galvao, D. S. Prediction of the hydrogen storage capacity of carbon nanoscrolls. Phys. Rev. B 2007, 75, 125404.

[20] Mpourmpakis, G.; Tylianakis, E.; Froudakis, G. E. Carbon nanoscrolls: A promising material for hydrogen storage. Nano Lett. 2007, 7, 1893-1897.

[21] Pan, H.; Feng, Y. P.; Lin, J. Y. Ab initio study of electronic and optical properties of multiwall carbon nanotube structures made up of a single rolled-up graphite sheet. Phys. Rev. B 2005, 72, 085415.

[22] Ordejon, P.; Artacho, E.; Soler, J. M. Self-consistent order-N density-functional calculations for very large systems. Phys. Rev. B 1996, 53, R10441-R10444.

[23] Soler, J. M.; Artacho, E.; Gale, J. D.; Garcia, A.; Junquera, J.; Ordejon, P.; Sanchez-Portal, D. The SIESTA method for ab initio order-N materials simulation. J. Phys.: Condens. Mat. 2002, 14, 2745-2779.

[24] Troullier, N.; Martins, J. L. Efficient pseudopotentials for plane-wave calculations. Phys. Rev. B 1991, 43, 19932006.

[25] Monkhorst, H. J.; Pack, J. D. Special points for Brillouinzone integrations. Phys. Rev. B 1976, 13, 5188-5192.

[26] Rocha, A. R.; Garcia-Suarez, V. M.; Bailey, S.; Lambert, C.; Ferrer, J.; Sanvito, S. Spin and molecular electronics in atomically generated orbital landscapes. Phys. Rev. B 2006, 73, 085414.

[27] Rocha, A. R.; Garcia-Suarez, V. M.; Bailey, S. W.; Lambert, C. J.; Ferrer, J.; Sanvito, S. Towards molecular spintronics. Nat. Mater. 2005, 4, 335-339.

[28] Martins, T. B.; da Silva, A. J. R.; Miwa, R. H.; Fazzio, A. oand $\pi$-defects at graphene nanoribbon edges: Building spin filters. Nano Lett. 2008, 8, 2293-2298.

[29] Gunlycke, D.; Areshkin, D. A.; Li, J. W.; Mintmire, J. W.; White, C. T. Graphene nanostrip digital memory device. Nano Lett. 2007, 7, 3608-3611.

[30] Enoki, T.; Kobayashi, Y. Magnetic nanographite: An approach to molecular magnetism. J. Mater. Chem. 2005, 15, 3999-4002.

[31] Enoki, T.; Takai, K. Unconventional electronic and magnetic functions of nanographene-based host-guest systems. Dalton Trans. 2008, 3773-3781. 